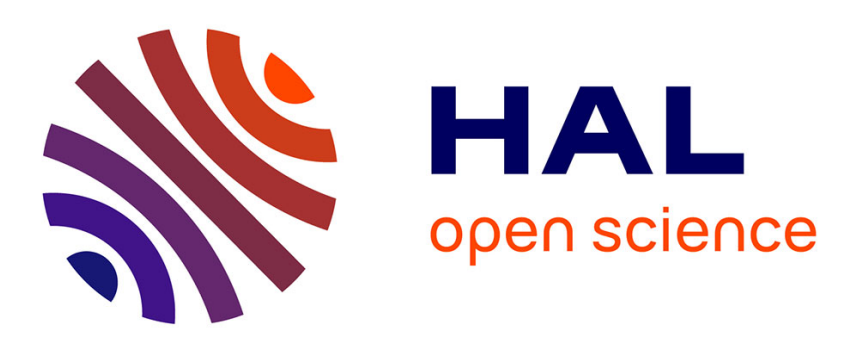

\title{
Casimir-Lifshitz Force Out of Thermal Equilibrium and Asymptotic Nonadditivity
}

Mauro Antezza, Lev P Pitaevskii, Sandro Stringari, Vitaly B Svetovoy

\section{To cite this version:}

Mauro Antezza, Lev P Pitaevskii, Sandro Stringari, Vitaly B Svetovoy. Casimir-Lifshitz Force Out of Thermal Equilibrium and Asymptotic Nonadditivity. Physical Review Letters, 2006, 97 (22), pp.223203. 10.1103/PhysRevLett.97.223203 . hal-02964025

\section{HAL Id: hal-02964025 \\ https://hal.science/hal-02964025}

Submitted on 12 Oct 2020

HAL is a multi-disciplinary open access archive for the deposit and dissemination of scientific research documents, whether they are published or not. The documents may come from teaching and research institutions in France or abroad, or from public or private research centers.
L'archive ouverte pluridisciplinaire HAL, est destinée au dépôt et à la diffusion de documents scientifiques de niveau recherche, publiés ou non, émanant des établissements d'enseignement et de recherche français ou étrangers, des laboratoires publics ou privés. 


\title{
Casimir-Lifshitz Force Out of Thermal Equilibrium and Asymptotic Nonadditivity
}

\author{
Mauro Antezza, ${ }^{1}$ Lev P. Pitaevskii, ${ }^{1,2}$ Sandro Stringari, ${ }^{1}$ and Vitaly B. Svetovoy ${ }^{3}$ \\ ${ }^{1}$ Dipartimento di Fisica, Università di Trento and CNR-INFM R\&D Center on Bose-Einstein Condensation, \\ Via Sommarive 14, I-38050 Povo, Trento, Italy \\ ${ }^{2}$ Kapitza Institute for Physical Problems, ul. Kosygina 2, 119334 Moscow, Russia \\ ${ }^{3}$ MESA+ Research Institute, University of Twente, PO 217, 7500 AE Enschede, The Netherlands
}

(Received 7 July 2006; published 30 November 2006)

\begin{abstract}
We investigate the force acting between two parallel plates held at different temperatures. The force reproduces, as limiting cases, the well-known Casimir-Lifshitz surface-surface force at thermal equilibrium and the surface-atom force out of thermal equilibrium recently derived by M. Antezza et al., Phys. Rev. Lett. 95, 113202 (2005). The asymptotic behavior of the force at large distances is explicitly discussed. In particular when one of the two bodies is a rarefied gas the force is not additive, being proportional to the square root of the density. Nontrivial crossover regions at large distances are also identified.
\end{abstract}

PACS numbers: $34.50 . D y, 12.20 .-\mathrm{m}, 42.50 . \mathrm{Vk}, 42.50 . \mathrm{Nn}$

The study of the thermal fluctuations of the electromagnetic field and of their effects on the force acting on surfaces and atoms is a long-standing subject of theoretical research starting from the seminal Lifshitz paper [1] (see also [2]). The elusive nature of the thermal component of the force follows from the fact that thermal effects becomes visible only at large distances, of the order of the photon thermal wave length $\lambda_{T}=\hbar c / k_{B} T$, where they prevail on the Casimir force originating from the $T=0$ quantum fluctuations of the field. At room temperature the thermal wavelength corresponds to about 7 microns, a distance at which both the Casimir and the thermal forces are very weak and difficult to reveal experimentally. The existence of thermal effects has been experimentally demonstrated only recently by the JILA experiment [3], by measuring the frequency shift of the center of mass motion of an ultracold atomic cloud located at a distance of a few microns from a dielectric substrate [4,5].

Thermal fluctuations determine the asymptotic, large distance behavior of the electromagnetic pressure which takes the Lifshitz form [2]

$$
P_{\mathrm{th}}^{\mathrm{eq}}(T, l)=\frac{k_{B} T}{16 \pi l^{3}} \int_{0}^{\infty} x^{2}\left[\frac{\left(\varepsilon_{10}+1\right)\left(\varepsilon_{20}+1\right)}{\left(\varepsilon_{10}-1\right)\left(\varepsilon_{20}-1\right)} e^{x}-1\right]^{-1} d x,
$$

in the case of two parallel surfaces separated by a distance $l$. Here $\varepsilon_{10}=\varepsilon_{1}(0)$ and $\varepsilon_{20}=\varepsilon_{2}(0)$ are the static dielectric constants of the two materials and $T$ is the temperature of the system. When the system is not in thermal equilibrium the pressure is expected to exhibit a different behavior. In particular, in recent papers the Trento team [6-8] has shown that the surface-atom force out of thermal equilibrium exhibits a new asymptotic behavior at large distances. With respect to that at equilibrium, the new force exhibits a slower dependence on the distance and a stronger dependence on the temperature, making its ex- perimental detection more accessible as demonstrated in the experiment of [3].

The purpose of the present work is to investigate the behavior of the force out of thermal equilibrium in the case of two parallel surfaces. The general goal is to better understand the role of thermal fluctuations which is not fully exploited at thermal equilibrium, being masked by peculiar cancellation effects between propagating and evanescent waves [6,7]. In particular, we address the following questions: (a) how is the Lifshitz law - and its asymptotic limit (1) - modified if the temperatures of the two bodies are different? (b) Can one recover the results of [6] for the surface-atom force when one body is made of a very dilute material corresponding to a gaseous phase with $(\varepsilon-1) \rightarrow 0$ ?

Let us consider two parallel dielectric half-spaces $l o$ cally at thermal equilibrium with different temperatures and separated by a distance $l$. In our configuration the leftside (right-side) body has a complex dielectric function $\varepsilon_{1}(\omega)\left[\varepsilon_{2}(\omega)\right]$ and is held at temperature $T_{1}\left(T_{2}\right)$, the whole system being in a stationary configuration. We assume that each body fills an infinite half-space [see, however, the discussion after Eq. (17)]. In practice this means that the bodies are thick compared to the penetration depth of the thermal radiation. In such conditions the presence of the remote surfaces of the bodies results only in a $l$-independent contribution to pressure, which will not be considered in this Letter.

Let us assume that the separation between the bodies is in the $z$ direction. Then the electromagnetic pressure between them is given by the average $P^{\text {neq }}\left(T_{1}, T_{2}, l\right)=$ $\left\langle T_{z z}(\mathbf{r}, t)\right\rangle$, where $T_{z z}(\mathbf{r}, t)=\left(E_{z}^{2}-E_{x}^{2}-E_{y}^{2}+B_{z}^{2}-B_{x}^{2}-\right.$ $\left.B_{y}^{2}\right) / 8 \pi$ is the $z z$ component of the Maxwell stress tensor [9] in the vacuum gap. In this work we focus on the thermal component $P_{\text {th }}$ of the force, defined by $P\left(T_{1}, T_{2}, l\right)=$ $P_{0}(l)+P_{\mathrm{th}}\left(T_{1}, T_{2}, l\right)$, where $P_{0}$ is the $T=0$ quantum 
pressure originating from the vacuum fluctuations of the field [2].

The electromagnetic field in the vacuum gap physically originates [9] from the fluctuating polarization fields $\mathbf{P}[\omega ; \mathbf{r}]$ in the two half-spaces whose correlations inside each body are described by the fluctuation-dissipation theorem

$$
\begin{aligned}
\left\langle P_{k}[\omega, \mathbf{r}] P_{l}^{*}\left[\omega^{\prime}, \mathbf{r}^{\prime}\right]\right\rangle= & \frac{\hbar \varepsilon_{1,2}^{\prime \prime}(\omega)}{2} \operatorname{coth}\left(\frac{\hbar \omega}{2 k_{B} T_{1,2}}\right) \\
& \times \delta\left(\omega-\omega^{\prime}\right) \delta\left(\mathbf{r}-\mathbf{r}^{\prime}\right) \delta_{k l},
\end{aligned}
$$

where $\varepsilon_{1,2}^{\prime \prime}(\omega)$ is the imaginary part of the dielectric function of the two materials. Because of the presence of the $\delta\left(\mathbf{r}-\mathbf{r}^{\prime}\right)$ factor these correlations are local [10] so that the effects of the fluctuations originating from the two halfspaces add incoherently. Assumption (2) (local source hypothesis) was first used in [11] and represents the starting point of our analysis allowing for an explicit calculation of the electromagnetic field also if the system is not in global thermal equilibrium $[6-8,12,13]$. The electric field at the point $\mathbf{r}$ in the gap can be in fact expressed in terms of the source polarization field via the convolution $\mathbf{E}[\omega ; \mathbf{r}]=$ $\int \mathbf{G}\left[\omega ; \mathbf{r}, \mathbf{r}^{\prime}\right] \cdot \mathbf{P}\left[\omega ; \mathbf{r}^{\prime}\right] d \mathbf{r}^{\prime}$ of the Green tensor [14], where the integration is performed over the volume containing the sources at $\mathbf{r}^{\prime}$. At the same time the magnetic field is easily evaluated using the Maxwell equation $\mathbf{B}[\omega ; \mathbf{r}]=-i \boldsymbol{\nabla} \times$ $\mathbf{E}[\omega ; \mathbf{r}] / k$, where $k=\omega / c$. Then Eq. (2) allows us to write the thermal pressure acting between the bodies as the sum of two terms:

$$
P_{\mathrm{th}}^{\mathrm{neq}}\left(T_{1}, T_{2}, l\right)=P_{\mathrm{th}}^{\mathrm{neq}}\left(T_{1}, 0, l\right)+P_{\mathrm{th}}^{\mathrm{neq}}\left(0, T_{2}, l\right),
$$

each of them corresponding to a configuration where only one of the two materials is at nonzero temperature. It is convenient to write

$$
\begin{aligned}
& P_{\mathrm{th}}^{\mathrm{neq}}(T, 0, l)=P_{\mathrm{th}}^{\mathrm{eq}}(T, l) / 2+\Delta P_{\mathrm{th}}(T, l), \\
& P_{\mathrm{th}}^{\mathrm{neq}}(0, T, l)=P_{\mathrm{th}}^{\mathrm{eq}}(T, l) / 2-\Delta P_{\mathrm{th}}(T, l),
\end{aligned}
$$

where $P_{\mathrm{th}}^{\mathrm{eq}}(T, l)$ is the Lifshitz pressure at equilibrium [2]. If we write the electromagnetic wave vector in the vacuum gap as $\mathbf{k}=\left(Q_{x}, Q_{y}, q_{z}\right)$, whose longitudinal part has modulus $Q=\sqrt{Q_{x}^{2}+Q_{y}^{2}}$, and $q_{z}=\sqrt{k^{2}-Q^{2}}$, it is possible to express the quantity $\Delta P_{\text {th }}$ as the sum $\Delta P_{\text {th }}=$ $\Delta P_{\mathrm{th}}^{\mathrm{PW}}+\Delta P_{\mathrm{th}}^{\mathrm{EW}}$, with

$$
\begin{gathered}
\Delta P_{\mathrm{th}}^{\mathrm{PW}}(T, l)=-\frac{\hbar}{4 \pi^{2}} \int_{0}^{\infty} d \omega \frac{1}{e^{\hbar \omega / k_{B} T}-1} \int_{0}^{k} d Q Q q_{z} \sum_{\mu=s, p}\left(\left|r_{2}^{\mu}\right|^{2}-\left|r_{1}^{\mu}\right|^{2}\right)\left(\frac{1}{\left|D_{\mu}\right|^{2}}-\frac{1}{1-\left|r_{1}^{\mu} r_{2}^{\mu}\right|^{2}}\right), \\
\Delta P_{\mathrm{th}}^{\mathrm{EW}}(T, l)=\frac{\hbar}{2 \pi^{2}} \int_{0}^{\infty} d \omega \frac{1}{e^{\hbar \omega / k_{B} T}-1} \int_{k}^{\infty} d Q Q \operatorname{Im} q_{z} e^{-2 l \operatorname{Im} q_{z}} \sum_{\mu=s, p} \frac{\operatorname{Im}\left(r_{1}^{\mu}\right) \operatorname{Re}\left(r_{2}^{\mu}\right)-\operatorname{Im}\left(r_{2}^{\mu}\right) \operatorname{Re}\left(r_{1}^{\mu}\right)}{\left|D_{\mu}\right|^{2}},
\end{gathered}
$$

where we have separated the effect of propagating waves (PW) from that of evanescent waves (EW) corresponding, respectively, to real and imaginary values of $q_{z}$. We have also subtracted the $l$-independent contributions so that both Eqs. (6) and (7) vanish as $l \rightarrow \infty$. In Eqs. (6) and (7) the dielectric properties of the two materials enters through the reflection Fresnel coefficients for the vacuum-dielectric interfaces

$$
r_{m}^{s}=\frac{q_{z}-q_{z}^{(m)}}{q_{z}+q_{z}^{(m)}}, \quad r_{m}^{p}=\frac{q_{z} \varepsilon_{m}-q_{z}^{(m)}}{q_{z} \varepsilon_{m}+q_{z}^{(m)}},
$$

where $s$ and $p$ correspond to the transverse electric and magnetic polarizations and $q_{z}^{(m)}=\sqrt{\varepsilon_{m} k^{2}-\bar{Q}^{2}}$ is the $z$ th component of the wave vector in the material $m=1,2$. The effect of multiple reflections between bodies is accounted for by the denominator $D_{\mu}=1-r_{1}^{\mu} r_{2}^{\mu} e^{2 i q_{z} l}$. It is evident that the pressure $P_{\text {th }}^{\text {neq }}(0, T, l)$ of Eq. (5) can be obtained from Eq. (4) by replacing $r_{1}^{\mu} \leftrightarrow r_{2}^{\mu}$. It is also immediate to see that Eq. (3) at thermal equilibrium $T_{1}=$ $T_{2} \equiv T$ provides the well-known equilibrium pressure $P_{\mathrm{th}}^{\mathrm{eq}}(T, l)$. For identical bodies $r_{1}^{\mu}=r_{2}^{\mu}$, yielding $\Delta P_{\mathrm{th}}=$ 0 , the pressure (3) is given by the expression

$$
P_{\mathrm{th}}^{\mathrm{neq}}\left(T_{1}, T_{2}, l\right)=P_{\mathrm{th}}^{\mathrm{eq}}\left(T_{1}, l\right) / 2+P_{\mathrm{th}}^{\mathrm{eq}}\left(T_{2}, l\right) / 2 .
$$

Equation (9), previously obtained in [15], is not, however, valid if the two materials are different. This is in disagreement with the results of [16], where (9) was found to be valid in general [17]. In particular Eqs. (4) and (5) show that interchanging the temperatures of the two materials implies a change in the force if the two materials are different. This clearly emerges from Fig. 1, where the results of a full calculation of the pressure between fused silica (body 1) and silicon (body 2) are presented [18]. It is interesting to note that the relative values of the pressure for the two nonequilibrium configurations (dashed and solid lines) strongly depends on the temperatures of the two bodies, on the separation $l$, and on the positions of the resonances of the two dielectric functions $\varepsilon_{1,2}(\omega)$. One can also see that both values are smaller than the one at thermal equilibrium (dotted line). This it is not, however, always the case. In fact, if one of the two bodies is rarefied the nonequilibrium pressure can become larger than at equilibrium (see [6] and discussion below).

In the following we will focus on the behavior of the force at large distances. For this study we will consider only dielectric bodies [where the limit $\varepsilon_{1,2}(\omega \rightarrow 0)$ is finite], while the case of metallic bodies will be presented elsewhere. The expansion of Eq. (4) results in the large 


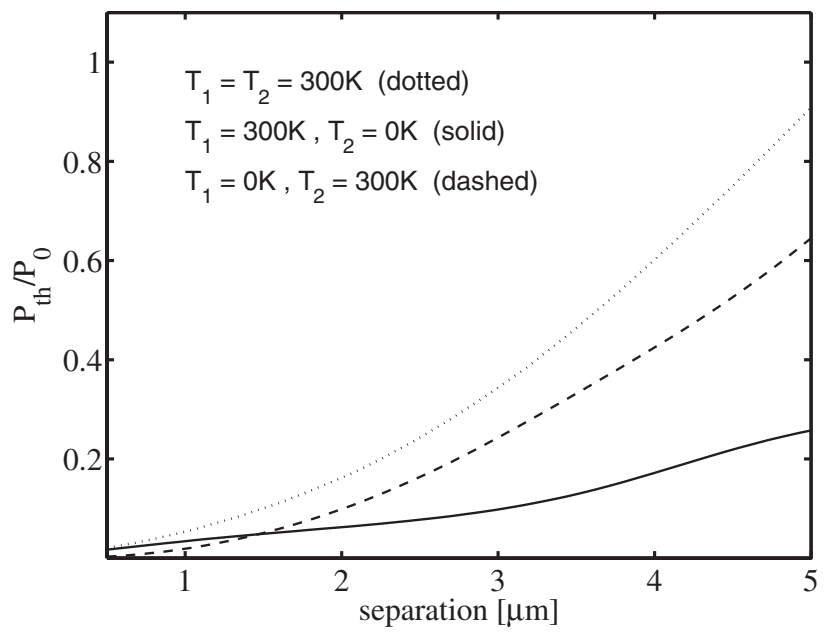

FIG. 1. Relative contribution of the thermal component with respect to the zero-temperature component of the pressure between two different materials: fused silica $\left(\mathrm{SiO}_{2}\right.$, body 1) and silicon ( $\mathrm{Si}$, body 2 ).

distance behaviors

$$
\begin{aligned}
P_{\mathrm{th}}^{\text {neq,PW }}(T, 0, l)= & \frac{k_{B} T}{l^{3}} \frac{\zeta(3)}{16 \pi}\left[2-\frac{\sqrt{\varepsilon_{10}-1}-\sqrt{\varepsilon_{20}-1}}{\sqrt{\varepsilon_{10}-1}+\sqrt{\varepsilon_{20}-1}}\right. \\
& \left.-\frac{\varepsilon_{20} \sqrt{\varepsilon_{10}-1}-\varepsilon_{10} \sqrt{\varepsilon_{20}-1}}{\varepsilon_{20} \sqrt{\varepsilon_{10}-1}+\varepsilon_{10} \sqrt{\varepsilon_{20}-1}}\right],
\end{aligned}
$$

$$
\begin{aligned}
P_{\mathrm{th}}^{\mathrm{neq}, \mathrm{EW}}(T, 0, l)= & \frac{k_{B} T}{l^{3}} \frac{1}{8 \pi^{2}} \int_{0}^{\infty} d t \int_{0}^{\infty} d x \frac{x^{2} e^{-x}}{t} \\
& \times \sum_{\mu=s, p} \frac{\operatorname{Im}\left[r_{1}^{\mu}(t)\right] \operatorname{Re}\left[r_{2}^{\mu}(t)\right]}{\left|1-r_{1}^{\mu}(t) r_{2}^{\mu}(t) e^{-x}\right|^{2}},
\end{aligned}
$$

holding for $l \gg \lambda_{T}$ [if one of the bodies is rarefied the condition becomes more stringent, see Eq. (14) below]. Here $\zeta(3)$ is the Riemann function, $r_{m}^{\mu}(t)$ are the Fresnel reflection coefficients calculated from Eq. (8) setting the static approximation $\varepsilon_{m}=\varepsilon_{m 0}$, and $Q^{2}=k^{2}\left(1+t^{2}\right)$. The PW and EW contributions of Eqs. (10) and (11) are of the same order but have opposite signs, the PW term being attractive and the EW repulsive. This feature is not, however, general and at shorter distance the signs of the PW and EW terms can change as discussed below. At distances of the order of the thermal wavelength Eqs. (10) and (11), as well as the Lifshitz result (1), provide only a crude estimate of the pressure. For example, in the case of the fused silica $\left(\varepsilon_{10} \approx 3.8\right)$-silicon $\left(\varepsilon_{20} \approx 11.7\right)$ configuration at $l=5 \mu \mathrm{m}$, the asymptotic laws overestimate the full calculation shown in Fig. 1 by a factor 1.5, 2, and 3 for the dashed, dotted, and solid lines, respectively.

Since in this work we are also interested in recovering the surface-atom force [6] which is relevant for the recent experiments with ultracold gases [3], it is useful to study the case in which the body 2 is cold and very rarefied, corresponding to small values of $\left(\varepsilon_{2}-1\right)=4 \pi n \alpha$. Here $n$ is the density of the material 2 and $\alpha$ is the dipole polarizability of the atoms. The expansion of pressure in Eq. (4) should be performed through two limiting procedures: the large distance $l \rightarrow \infty$ and the diluteness $\left(\varepsilon_{2}-1\right) \rightarrow 0$ conditions. It is crucial to identify the proper order of the two limits.

One relevant asymptotic behavior is obtained by first taking the limit of large $l$ at fixed $\varepsilon_{2}$ [this yields, by the way, expressions (10) and (11)], and then carrying out the limit of rarefied body. One finds the expression

$$
P_{\mathrm{th}}^{\mathrm{neq}}(T, 0, l)=\frac{k_{B} T C}{l^{3}} \frac{\varepsilon_{10}+1}{\sqrt{\varepsilon_{10}-1}} \sqrt{\varepsilon_{20}-1},
$$

for the total pressure, where $C=C_{\mathrm{PW}}+C_{\mathrm{EW}} \approx 3.83 \times$ $10^{-2}$, is a numerical factor with $C_{\mathrm{PW}}=\zeta(3) / 8 \pi \approx$ $4.78 \times 10^{-2}$ and $C_{\mathrm{EW}} \approx-0.96 \times 10^{-2}$.

The peculiar $\sqrt{\varepsilon_{20}-1}$ dependence of (12) means that the pressure acting on the atoms of the substrate 2 is not additive. Additivity would in fact require a linear dependence on $\left(\varepsilon_{20}-1\right)$, and hence on the gas density $n$, as happens for the Lifshitz pressure (1) as $\left(\varepsilon_{20}-1\right) \rightarrow 0$ :

$$
P_{\mathrm{th}}^{\mathrm{eq}}(T, l)=\frac{k_{B} T}{16 \pi l^{3}} \frac{\varepsilon_{10}-1}{\varepsilon_{10}+1}\left(\varepsilon_{20}-1\right) .
$$

The nonadditivity of the pressure (12) follows from the fact that for large $l$ the main contribution in the force is produced by the grazing waves incident on the interface of the material 2 from the vacuum gap at small angles corresponding to small values of $q_{z} / k \leq \sqrt{\varepsilon_{20}-1}$. Hence the reflection coefficients from the body 2 are not small even at small $\varepsilon_{20}-1$ and the body cannot be considered dilute from the electrodynamic point of view. This is a peculiarity of the nonequilibrium situation. In fact, at equilibrium this anomalous contribution is canceled by the waves impinging the interface from the interior of the dielectric 2, close to the angle of total reflection. Notice that result (12) is valid at the condition

$$
l \gg \lambda_{T} / \sqrt{\varepsilon_{20}-1},
$$

which becomes stronger and stronger as $\left(\varepsilon_{20}-1\right) \rightarrow 0$.

The second limiting procedure is obtained by first taking the expansion of (4) for small values of $\left(\varepsilon_{2}-1\right)$, and then carrying out the limit of large distances. In this case the relevant wave vectors satisfy the condition $q_{z} / k \gg$ $\sqrt{\varepsilon_{20}-1}$, and the PW component produces a contribution identical to the EW one, yielding

$$
P_{\text {th }}^{\text {neq }}(T, 0, l)=\frac{\left(k_{B} T\right)^{2}}{24 l^{2} c h} \frac{\varepsilon_{10}+1}{\sqrt{\varepsilon_{10}-1}}\left(\varepsilon_{20}-1\right) .
$$

Result (15) holds in the distance range complementary to (14)

$$
\lambda_{T} \ll l \ll \lambda_{T} / \sqrt{\varepsilon_{20}-1} .
$$

In deriving Eq. (15) we also replaced $\varepsilon_{1,2}(\omega)$ with their static values $\varepsilon_{m 0}$. This is justified if $k_{B} T$ is much smaller 


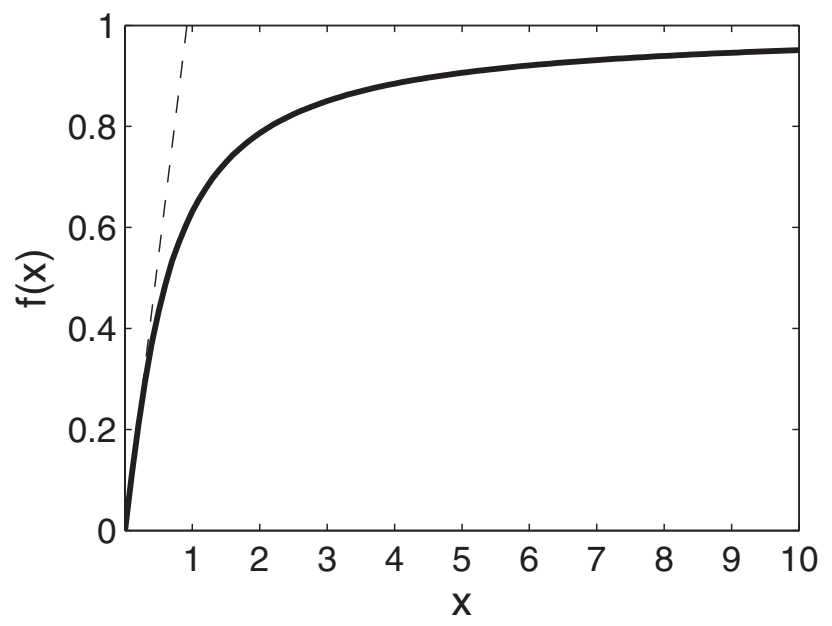

FIG. 2. Dimensionless function $f(x)$ [see Eq. (17)]. The dashed line gives the asymptotic limit at small $x$.

than the lowest resonances of both the body 1 and the atoms of the dilute body 2. It is worth noting that the interval (16) practically disappears for dense dielectrics.

It is also worth noticing that, due to the diluteness condition $\left(\varepsilon_{20}-1\right) \ll 1$ and as a consequence of Eqs. (4) and (5), in both regions (14) and (16) the term $\Delta P_{\text {th }}$ gives the leading contribution to the pressure and consequently the $l$-dependent interaction between the two bodies will be attractive if $T_{1}>T_{2}$ and repulsive in the opposite case.

The transition between the two regimes (12) and (15) can be investigated performing the diluteness limit $\left(\varepsilon_{20}-\right.$ 1) $\rightarrow 0$ in Eq. (4), for a fixed value of the dimensionless variable $x=l \sqrt{\varepsilon_{20}}-1 / \lambda_{T}$. The results are reported in Fig. 2, where the thermal pressure $P_{\mathrm{th}}^{\text {neq }}(T, 0, l)$ is plotted in units of the asymptotic behavior (12):

$$
P_{\mathrm{th}}^{\mathrm{neq}}(T, 0, l)=\frac{k_{B} T C}{l^{3}} \frac{\varepsilon_{10}+1}{\sqrt{\varepsilon_{10}-1}} \sqrt{\varepsilon_{20}-1} f(x) .
$$

Here $f(x)$ is a dimensionless function of the variable $x$. When $x \rightarrow \infty$ [regime (12)] one has $f(x) \rightarrow 1$, while when $x \rightarrow 0$ [regime (15)] one finds $f(x) \rightarrow x / 24 C \approx 1.09 x$.

In order to recover the asymptotic result of [6] for the surface-atom force out of thermal equilibrium it is crucial to follow the second limiting procedure, leading to result (15). In this case, however, the PW term must be omitted since the atomic gas occupies a finite region of space and does not absorb the thermal radiation. Using the formalism of the present work this corresponds to treating the body 2 as a slab of rarefied gas of thickness $L$ for which one should also take into account the force acting on its remote surface. In the absence of absorption [19] it is possible to show that, including refraction at the remote surface, the PW pressure becomes vanishingly small, of order $\left(\varepsilon_{20}-1\right)^{2}$ with respect to Eq. (15). In this case the EW contribution, which is $1 / 2$ of (15), provides the total pressure acting on the gas and is exactly equivalent to Eq. (12) of [6] for the surface-atom force. Notice that in the derivation of [6] the leading role of the EW term was stressed from the very beginning.

In conclusion, we have generalized the Casimir-Lifshitz surface-surface force to configurations out of thermal equilibrium and calculated the corresponding asymptotic behavior. When one of the two bodies is a rarefied gas a crossover region emerges where the pressure changes from a $T^{2} / l^{2}$ behavior, characterizing the surface-atom interaction, to a region at very large distances where the pressure behaves like $T / l^{3}$ and is no longer additive.

We gratefully acknowledge G. Carugno and E. Cornell for stimulating discussions, and I. Dorofeyev for a critical reading of the Letter. We also acknowledge supports by the Ministero dell'Istruzione, dell'Università e della Ricerca (MIUR).

[1] E. M. Lifshitz, Dokl. Akad. Nauk SSSR 100, 879 (1955).

[2] I. E. Dzyaloshinskii, E. M. Lifshitz, and L. P. Pitaevskii, Adv. Phys. 10, 165 (1961).

[3] J.M. Obrecht, R. J. Wild, M. Antezza, L. P. Pitaevskii, S. Stringari, and E. A. Cornell, physics/0608074 [Phys. Rev. Lett. (to be published)].

[4] M. Antezza, L. P. Pitaevskii, and S. Stringari, Phys. Rev. A 70, 053619 (2004).

[5] D. M. Harber, J. M. Obrecht, J. M. McGuirk, and E. A. Cornell, Phys. Rev. A 72, 033610 (2005).

[6] M. Antezza, L. P. Pitaevskii, and S. Stringari, Phys. Rev. Lett. 95, 113202 (2005).

[7] L. P. Pitaevskii, J. Phys. A 39, 6665 (2006).

[8] M. Antezza, J. Phys. A 39, 6117 (2006).

[9] L.D. Landau and E. M. Lifshitz, Electrodynamics of Continuous Media (Pergamon, New York, 1963).

[10] The presence of the delta function is justified by the fact that the relevant distances are much larger than the nonlocality lengths (nanometers) characterizing the optical properties of the media.

[11] D. Polder and M. Van Hove, Phys. Rev. B 4, 3303 (1971).

[12] K. Joulain, J.-P. Mulet, F. Marquier, R. Carminati, and J.-J. Greffet, Surf. Sci. Rep. 57, 59 (2005).

[13] C. Henkel, K. Joulain, J.-P. Mulet, and J.-J. Greffet, J. Opt. A Pure Appl. Opt. 4, S109 (2002).

[14] J. E. Sipe, J. Opt. Soc. Am. B 4, 481 (1987); M. S. Tomas, Phys. Rev. A 66, 052103 (2002).

[15] I. A. Dorofeyev, J. Phys. A 31, 4369 (1998).

[16] I. Dorofeyev, H. Fuchs, and J. Jersch, Phys. Rev. E 65, 026610 (2002).

[17] After online submission of the present Letter, I. Dorofeyev informed us that the general formalism developed in [15] agrees with Eqs. (4)-(8), so that we argue that results of [16] were based on some inconsistent derivation.

[18] We used the experimental values of $\varepsilon_{1,2}(\omega)$ taken from W. J. Tropf and M.E. Thomas, in Handbook of Optical Constants of Solids, edited by E. Palik (Academic, New York, 1998), Vol. III.

[19] Neglecting the absorption actually requires the condition $L \ll \lambda_{T}^{2} / l \varepsilon_{2}^{\prime \prime}$. We also assume that there is no thermal radiation coming from $z=+\infty$. 